\title{
NONNUCLEAR SUBALGEBRAS OF AF ALGEBRAS
}

\author{
MARIUS DADARLAT
}

\begin{abstract}
We show that any non type I separable unital AF algebra $B$ can be modelled from inside by a nonnuclear $\mathrm{C}^{*}$-algebra and from outside by a nonexact $\mathrm{C}^{*}$-algebra. More precisely there exist unital separable quasidiagonal $\mathrm{C}^{*}$-algebras $A \subset B \subset C$ of real rank zero, stable rank one, such that $A$ is nonnuclear, $C$ is nonexact, and both $A$ and $C$ are asymptotically homotopy equivalent to $B$. In particular $A, B$ and $C$ have the same ordered K-theory groups, hence isomorphic ideal lattices, and, $A$ and $B$ have (affinely) homeomorphic trace spaces.
\end{abstract}

\section{Introduction}

The type $\mathrm{I} \mathrm{C}^{*}$-algebras are nuclear $\left[\mathrm{Ta}_{1}\right]$. Every $\mathrm{C}^{*}$-subalgebra of a type $\mathrm{I} \mathrm{C}^{*}$ algebra is type $\mathrm{I}$ hence nuclear. On the other hand Blackadar showed that any nontype $\mathrm{I} \mathrm{C}^{*}$-algebra contains a nonnuclear $\mathrm{C}^{*}$-subalgebra $\left[\mathrm{B}_{1}\right]$. Thus a $\mathrm{C}^{*}$-algebra is type $\mathrm{I}$ if and only if all its $\mathrm{C}^{*}$-subalgebras are nuclear.

The main result of this paper shows that every non type I unital AF algebra $B$ contains a nonnuclear unital $\mathrm{C}^{*}$-subalgebra $A$ and is contained in a unital nonexact C*$^{*}$-algebra $C$ such that both $A$ and $C$ "model" $B$ (see Proposition 9 and Theorem $11)$.

In [Ha], Hadwin raises the question of whether every strongly quasidiagonal $\mathrm{C}^{*}$ algebra is nuclear. In $[\mathrm{R}]$, Rosenberg shows that if the reduced $\mathrm{C}^{*}$-algebra of a discrete countable group is quasidiagonal then $G$ is amenable, and asks whether the strongly quasidiagonal $\mathrm{C}^{*}$-algebras are exact.

The examples of nonnuclear quasidiagonal $\mathrm{C}^{*}$-subalgebras of non type I AF algebras exhibited in $\left[\mathrm{B}_{1}\right]$ are not strongly quasidiagonal since they have a nonquasidiagonal quotient, the Choi algebra. They are also nonsimple, as noticed in [Po]. Let $\mathcal{C}$ be the class of all separable simple unital quasidiagonal real rank zero $\mathrm{C}^{*}$-algebras with unique unital trace. Popa pointed out that Connes' Følner type condition for a $\mathrm{II}_{1}$ factor $M$ (which implies that $M$ is hyperfinite [C, p.501]), coincides with the Hilbert-Schmidt-norm version of the local finite dimensional approximation property that characterizes the quasidiagonality of a simple $\mathrm{C}^{*}$-algebra. Actually, using an adaptation to the $\mathrm{C}^{*}$-algebra context of his short proof of Connes' theorem

This research was partially supported by the National Science Foundation, \# 9622434-DMS. 
of equivalence between injectiveness and hyperfinitness, Popa has shown that any $\mathrm{C}^{*}$-algebra $A$ in the class $\mathcal{C}$ satisfies the following local finite dimensional approximation property. For any finite subset $F$ of $A$ and any $\epsilon>0$, there is a nonzero finite dimensional matrix subalgebra $C$ of $A$ with unit $e$ such that $\|e x-x e\|<\epsilon$ and $\operatorname{dist}(x, C)<\epsilon$ for all $x \in F$. The question of whether this local approximation could be extended to a suitable global approximation that would imply the nuclearity of $A$ was left open. In this context, Popa has asked whether every $\mathrm{C}^{*}$-algebra in $\mathcal{C}$ is nuclear.

We answer Popa's question by showing that $\mathcal{C}$ contains $\mathrm{C}^{*}$-algebras which are not exact (or subnuclear), and exact $\mathrm{C}^{*}$-algebras which are not nuclear. Since any simple quasidiagonal $\mathrm{C}^{*}$-algebra is strongly quasidiagonal, this also yields a negative answer to the questions of Hadwin and Rosenberg.

In the very last part of the paper, Example 13, (which is joint work with Chris Phillips), we exhibit two nonisomorphic separable simple exact $\mathrm{C}^{*}$-algebras of real rank zero and stable rank one, with the same Elliott and Haagerup invariants. Real rank one examples with similar properties have recently been exhibited in [GePh].

Thanks are due to the referee for a number of suggestions that improved the exposition.

\section{The $\mathrm{C}^{*}$-algebra of a Bratteli system}

All the $\mathrm{C}^{*}$-algebras in this paper are assumed to be separable. The identity map of $M_{r}(\mathbb{C})$ will be denoted by $i d_{r}$.

Definition 1. A Bratteli system $(A, \underline{\pi})$ consists of a unital $\mathrm{C}^{*}$-algebra $A$ and a sequence $\underline{\pi}=\left(\pi_{n}\right)_{n=1}^{\infty}$ where each $\pi_{n}=\left(\pi_{n}^{i, j}\right), 1 \leq i \leq t_{n+1}, 1 \leq j \leq t_{n}$ is a $t_{n+1} \times t_{n}$ matrix of unital $*$-homomorphisms

$$
\pi_{n}^{i, j}: A \rightarrow M_{k_{n}^{i, j}}(A), \quad k_{n}^{i, j} \in \mathbb{N} .
$$

We always assume that $t_{1}=1$.

There is an inductive system $\left(A_{n}, \phi_{n}\right)$ associated in a canonical way to a Bratteli system. For every $n \in \mathbb{N}$ define integers $[n, i], 1 \leq i \leq t_{n}$, recursively by

$$
[n+1, i]=\sum_{j=1}^{t_{n}}[n, j] k_{n}^{i, j}, \quad 1 \leq i \leq t_{n+1}, \quad[1,1]=1 .
$$

For every $n \geq 1$ let $A_{n}^{i}=M_{[n, i]}(A)$, and

$$
A_{n}=\bigoplus_{i=1}^{t_{n}} A_{n}^{i}=\bigoplus_{i=1}^{t_{n}} M_{[n, i]}(A)
$$

Let $\phi_{n}: A_{n} \rightarrow A_{n+1}$ be the unital $*$-homomorphism given by a matrix $\left(\phi_{n}^{i, j}\right)$ of partial $*$-homomorphisms, where

$$
\phi_{n}^{i, j}=i d_{[n, j]} \otimes \pi_{n}^{i, j}: M_{[n, j]}(A) \rightarrow M_{[n, j] k_{n}^{i, j}}(A) \subset M_{[n+1, i]}(A) .
$$


The corestriction of $\phi_{n}$ to $M_{[n+1, i]}(A)$, denoted by $\phi_{n}^{i}$, maps $a=\left(a_{1}, \ldots, a_{t_{n}}\right) \in A_{n}$ to $\phi_{n}^{i}(a)=\oplus_{j=1}^{t_{n}} \phi_{n}^{i, j}\left(a_{j}\right)$. Note that $A_{1}=A$ since $t_{1}=1$. If $n<m$ we set

$$
\phi_{m, n}=\phi_{m-1} \circ \cdots \circ \phi_{n}: A_{n} \rightarrow A_{m}
$$

and in particular $\phi_{n+1, n}=\phi_{n}$.

Definition 2. The $C^{*}$-algebra of a Bratteli system $(A, \underline{\pi})$ is defined by $A(\underline{\pi})=\underline{\lim _{1}}\left(A_{n}, \phi_{n}\right)$.

The induced map $A_{n} \rightarrow A(\underline{\pi})$ is denoted by $\phi_{\infty, n}$. The definition of $A(\underline{\pi})$ was inspired by similar constructions involving homogeneous $\mathrm{C}^{*}$-algebras, and in particular by $[\mathrm{Go}]$. Note that any Bratteli system $(A, \underline{\pi})$ determines a Bratteli diagram (see $[\mathrm{Br}]$ ) with incidence matrices $\left(k_{n}^{i, j}\right)$. The corresponding AF algebra is denoted by $A F(\underline{\pi})$.

Definition 3. A Bratteli system $(A, \underline{\pi})$ is called restricted if the following two conditions are satisfied for every $n \geq 1$.

(i) If $(i, j) \neq(1,1)$, then $\pi_{n}^{i, j}$ is a finite dimensional unital representation $\pi_{n}^{i, j}: A \rightarrow M_{k_{n}^{i, j}}(\mathbb{C})$, which is to be regarded as a map into $M_{k_{n}^{i, j}}(A)$ by identifying $M_{k_{n}^{i, j}}(\mathbb{C})$ with $M_{k_{n}^{i, j}}\left(\mathbb{C} 1_{A}\right)$.

(ii) $\pi_{n}^{1,1}: A \rightarrow M_{k_{n}^{1,1}}(A)$ is of the form

$$
\pi_{n}^{1,1}(a)=\left(\begin{array}{cc}
a & 0 \\
0 & \gamma_{n}(a)
\end{array}\right)
$$

where $\gamma_{n}: A \rightarrow M_{k(n)}\left(\mathbb{C} 1_{A}\right)$ is a finite dimensional unital representation, and $k(n)=k_{n}^{1,1}-1$. The sequence $\left(\gamma_{n}\right)_{n=1}^{\infty}$ will be called the distinguished component of $\underline{\pi}$.

Let $A$ be a separable $C^{*}$-algebra and let $\left(\gamma_{n}\right)_{n=1}^{\infty}$ be a sequence of finite dimensional representations of $A$. We say that $\left(\gamma_{n}\right)_{n=1}^{\infty}$ is a separating sequence if for every $a \in A$ there is $n$ such that $\gamma_{n}(a) \neq 0$. If this the case we say that $A$ is residually finite dimensional (abbreviated RFD). A sequence $\left(\gamma_{n}\right)_{n=1}^{\infty}$ is called strongly separating if for every $a \in A$, and any $m \geq 1$, there is $n \geq m$ such that $\gamma_{n}(a) \neq 0$. We say that $\left(\gamma_{n}\right)_{n=1}^{\infty}$ has infinite multiplicity if each representation in the sequence repeats itself infinitely many times. It is clear that if $\left(\gamma_{n}\right)_{n=1}^{\infty}$ is separating and has infinite multiplicity, then $\left(\gamma_{n}\right)_{n=1}^{\infty}$ is strongly separating. Note that for any unital separable RFD C*-algebra there exist restricted Bratteli systems whose distinguished component $\left(\gamma_{n}\right)_{n=1}^{\infty}$ is strongly separating,

Remark 4. The construction $A(\underline{\pi})$ has a certain functorial property. Suppose that $B$ is a unital $\mathrm{C}^{*}$-subalgebra of $A$, with $1_{B}=1_{A}$, and such that $\pi_{n}^{i, j}(B) \subset M_{k_{n}^{i, j}}(B)$ for all $\pi_{n}^{i, j}$. If $\iota_{0}: B \rightarrow A$ denotes the inclusion map, then all the diagrams

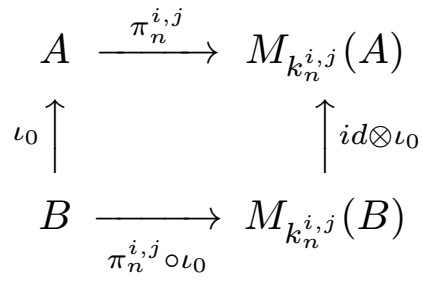


are commutative. It is then clear that $\left(B, \underline{\pi} \circ \iota_{0}\right)$ is a Bratteli system and $\iota_{0}$ induces a unital $*$-monomorphism $\iota: B\left(\underline{\pi} \circ \iota_{0}\right) \rightarrow A(\underline{\pi})$.

Note that if $(A, \underline{\pi})$ is a restricted Bratteli system, then the above diagram commutes for any unital $\mathrm{C}^{*}$-subalgebra $B$ of $A$, with $1_{B}=1_{A}$. In this case $\left(B, \underline{\pi} \circ \iota_{0}\right)$ is also a restricted Bratteli system. Note that if $\left(\gamma_{n}\right)_{n=1}^{\infty}$ is (strongly ) separating, then so is $\left(\gamma_{n} \circ \iota_{0}\right)_{n=1}^{\infty}$.

In particular if $B=\mathbb{C} 1_{A}, \iota_{0}: \mathbb{C} \cong \mathbb{C} 1_{A} \hookrightarrow A$, then $A F(\underline{\pi}) \cong \mathbb{C}\left(\underline{\pi} \circ \iota_{0}\right) \hookrightarrow A(\underline{\pi})$.

The notions of (topological) stable rank (sr) and real rank (RR) of a $\mathrm{C}^{*}$-algebra were defined in $[\mathrm{BP}]$ and respectively $[\mathrm{Ri}]$.

Proposition 5. Let $(A, \underline{\pi})$ be a restricted Bratteli system. If the sequence $\left(\gamma_{n}\right)_{n=1}^{\infty}$ is strongly separating, then $A(\underline{\pi})$ has real rank zero and stable rank one.

Proof. We prove first that $\operatorname{RR}(A)=0$. Since the finite dimensional $\mathrm{C}^{*}$-algebras have real rank zero, and since $\phi_{n}^{i}$ has finite dimensional image for $i \neq 1$, it suffices to show that for every $n \geq 1$, every $\epsilon>0$, and every noninvertible element $a=$ $a^{*} \in A_{n}$, there is $m>n$ and $b=b^{*} \in G L\left(A_{m}^{1}\right)$ such that $\left\|\phi_{m, n}^{1}(a)-b\right\|<\epsilon$. After replacing $a$ by $\phi_{n}(a)$ and after slightly perturbing the scalar components of $\phi_{n}(a)$, we may assume that if $a=\left(a_{1}, \ldots, a_{t_{n}}\right)$, then $a_{i}$ is invertible in $A_{n}^{i}$ for $i \neq 1$ and $x=x^{*}=a_{1}$ is not invertible in $A_{n}^{1}$. Fix $n, \epsilon$ and $a \in A_{n}$ as above and let $\sigma_{m}=i d_{[n, 1]} \otimes \pi_{m}: M_{[n, 1]}(A) \rightarrow M_{[n, 1] k(m)}(\mathbb{C})$ for all $m>n$. Then $\left(\sigma_{m}\right)_{m>n}$ is a separating sequence of unital representations for $A_{n}^{1}$, hence

$$
\sigma=\sigma_{n+1} \oplus \sigma_{n+2} \oplus \ldots
$$

is a unital faithful representation of $A_{n}^{1}$. Since $\sigma(x)$ is necessarily noninvertible, it follows that if $x_{m}=\sigma_{m}(x)$ then either $(i)$ there is $m>n$ such that $x_{m}$ is not invertible, or $($ ii $) x_{m}$ is invertible for all $m>n$ and $\overline{\lim }_{m \rightarrow \infty}\left\|x_{m}^{-1}\right\|=\infty$. Now $x_{m}$ is a selfadjoint element in $M_{[n, 1] k(m)}(\mathbb{C})$. Thus there is a unitary $u_{m}$ such that

$$
x_{m}=u_{m}\left(\begin{array}{cc}
\lambda_{m} 1_{A} & 0 \\
0 & y_{m}
\end{array}\right) u_{m}^{*}
$$

where $\lambda_{m}$ is an eigenvalue of $x_{m}$ of minimum absolute value and $y_{m}=y_{m}^{*} \in$ $M_{[n, 1] k(m)-1}(\mathbb{C})$. Note that in case $(i)$ there is $m>n$ such that $\lambda_{m}=0$, and in case $(i i)$ there is $m>n$ such that $\left|\lambda_{m}\right|<\epsilon$, since the sequence $\left\|x_{m}^{-1}\right\|=\left|\lambda_{m}\right|^{-1}$ is unbounded. Thus we can always find $m>n$ such that $\left|\lambda_{m}\right|<\epsilon$. Note that up to a conjugation by a unitary, $\phi_{m-1, n}^{1}(a)$ and $\phi_{m, n}^{1}(a)$ are of the form

$$
\left(\begin{array}{ll}
x & 0 \\
0 & y
\end{array}\right) \text {, and respectively }\left(\begin{array}{ccc}
x & 0 & 0 \\
0 & x_{m} & 0 \\
0 & 0 & z
\end{array}\right)
$$

where $y=y^{*}$ and $z=z^{*}$ are matrices over $\mathbb{C} 1_{A}$. Therefore there is a unitary $v \in A_{m}^{1}$ such that

$$
\phi_{m, n}^{1}(a)=v\left(\begin{array}{ccc}
x & 0 & 0 \\
0 & \lambda_{m} 1_{A} & 0 \\
0 & 0 & c
\end{array}\right) v^{*}
$$


where $c$ is a selfadjoint matrix over $\mathbb{C} 1_{A}$. Let $\delta$ be such that $0<\delta<\epsilon-\left|\lambda_{m}\right|$ and $c+\delta$ is invertible. Then

$$
b=b^{*}=v\left(\begin{array}{ccc}
x & \delta & 0 \\
\delta & 0 & 0 \\
0 & 0 & c+\delta
\end{array}\right) v^{*} \in G L\left(A_{m}^{1}\right)
$$

and $\left\|\phi_{m, n}^{1}(a)-b\right\| \leq\left|\lambda_{m}\right|+\delta<\epsilon$.

Next we show that $\operatorname{sr}(A)=1$. Reasoning as in the first part of the proof, it suffices to show that for every $n \geq 1$, every $\epsilon>0$ and every element $a=$ $\left(a_{1}, \ldots, a_{t_{n}}\right) \in A_{n}$, whose only noninvertible component is $x=a_{1}$, there is $m>n$ and $b \in G L\left(A_{m}^{1}\right)$ such that $\left\|\phi_{m, n}^{1}(a)-b\right\|<\epsilon$. Let $\sigma_{m}$ and $\sigma$ be as in the first part of the proof. Using the polar decomposition of $\sigma_{m}(x)$, we can write $\sigma_{m}(x)=w_{m} x_{m}$, where $w_{m}$ is a unitary and $x_{m} \geq 0$. Since $\sigma(x)$ is not invertible, the sequence $\left(x_{m}\right)_{m>n}$ must verify either $(i)$ or $(i i)$. Reasoning as above, we find $m>n$ and unitaries $u, v \in A_{m}^{1}$ such that

$$
\phi_{m, n}^{1}(a)=u\left(\begin{array}{ccc}
x & 0 & 0 \\
0 & \lambda_{m} 1_{A} & 0 \\
0 & 0 & c
\end{array}\right) v
$$

where $\left|\lambda_{m}\right|<\epsilon$ and $c$ is some matrix over $\mathbb{C} 1_{A}$. We complete the proof by taking

$$
b=u\left(\begin{array}{ccc}
x & \delta & 0 \\
\delta & 0 & 0 \\
0 & 0 & c+\delta
\end{array}\right) v \in G L\left(A_{m}^{1}\right)
$$

with $0<\delta<\epsilon-\left|\lambda_{m}\right|$ and $c+\delta$ invertible.

Proposition 6. Let $(A, \underline{\pi})$ be a restricted Bratteli system.

(i) $A(\underline{\pi})$ is exact if and only if $A$ is exact.

(ii) $A(\underline{\pi})$ is nuclear if and only if $A$ is nuclear.

Proof. (i) If $A$ is exact, then $A(\underline{\pi})$ is exact being an inductive limit of the exact $\mathrm{C}^{*}$-algebras $A_{n}=\oplus_{i} M_{[n, i]}(A)[\mathrm{W}, 2.5 .5]$. Conversely if $A(\underline{\pi})$ is exact, then $A$ is exact as it embeds in $A(\underline{\pi})$ [W, Proposition 2.6].

(ii) If $A$ is nuclear, then $A(\underline{\pi})$ is nuclear being an inductive limit of nuclear $\mathrm{C}^{*}$ algebras. Conversely, suppose that $A(\underline{\pi})$ is nuclear. Let $F \subset A_{1}=A$ be a finite subset and let $\epsilon>0$. Let $\phi_{\infty, n}: A_{n} \rightarrow A(\underline{\pi})$ be the induced map. Since $A(\underline{\pi})$ is nuclear, the map $\phi_{\infty, 1}$ is nuclear, hence there exist a finite dimensional $\mathrm{C}^{*}$-algebra $C$ and unital completely positive maps $\alpha: A_{1} \rightarrow C$ and $\beta: C \rightarrow A(\underline{\pi})$ such that

$$
\left\|\phi_{\infty, n}(a)-\beta \alpha(a)\right\|<\epsilon / 2
$$

for all $a \in F$. By [DL, Lemma 4.2] (an application of the Choi-Effros lifting Theorem [ChE]), we find $n \geq 1$ and a unital completely positive map $\beta_{n}: C \rightarrow A_{n}$ such that

$$
\left\|\beta(x)-\phi_{\infty, n} \beta_{n}(x)\right\|<\epsilon / 2
$$


for all $x \in \alpha(F)$. From (1) and (2) we obtain

$$
\left\|\phi_{\infty, n}\left(\phi_{n, 1}(a)-\beta_{n} \alpha(a)\right)\right\| \leq\left\|\phi_{\infty, n}(a)-\beta \alpha(a)\right\|+\left\|\beta \alpha(a)-\phi_{\infty, n} \beta_{n} \alpha(a)\right\|<\epsilon
$$

for all $a \in F$. Since $\phi_{\infty, n}$ is isometric, it follows that

$$
\left\|\phi_{n, 1}(a)-\beta_{n} \alpha(a)\right\|<\epsilon
$$

for all $a \in F$. Let $e=1_{A} \oplus 0_{[n, 1]-1}$ be the projection in $A_{n}^{1}=M_{[n, 1]}(A)$ corresponding to the $(1,1)$ corner. We have $e \phi_{n, 1}(a) e=a$, hence if $\eta_{n}=e(-) e: A_{n} \rightarrow A$, then from (3), we obtain $\left\|a-\eta_{n} \beta_{n} \alpha(a)\right\|<\epsilon$ for all $a \in F$. This proves that $A$ is nuclear. The flow of the proof is illustrated by the following diagram.

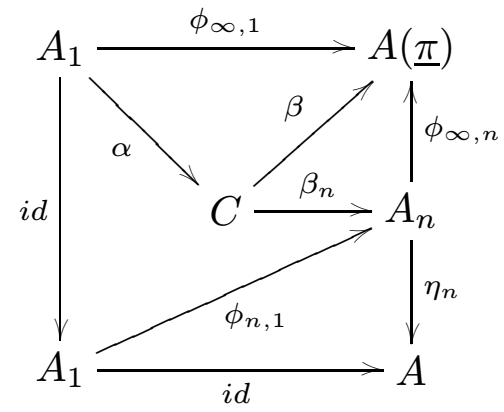

\section{Nonexact models of AF algebras}

Lemma 7. Let $B$ be a non type I unital AF algebra. Let $(k(n))_{n=1}^{\infty}$ be a sequence of integers. Then there is an inductive system $\left(B_{n}, \psi_{n+1, n}\right)$ with limit isomorphic to $B$, where $B_{n}$ are finite dimensional $C^{*}$-algebras, $B_{1}=\mathbb{C}, \psi_{n+1, n}$ are unital $*$ homomorphisms given by incidence matrices $\left(k_{n}^{i, j}\right), 1 \leq i \leq t_{n+1}, 1 \leq j \leq t_{n}$, and $k_{n}^{1,1}>k(n)$ for all $n \geq 1$.

Proof. By [Pe, p.191], if $B$ is not type I, then $B$ has a quotient $D$ without nonzero abelian elements, i.e. $D$ is antiliminary. By Theorem 3.3 of $[\mathrm{Br}]$, any nonzero quotient of $B$ admits a Bratteli diagram given by a full subgraph of some Bratteli diagram of $B$. Therefore it suffices to prove the statement for some nonzero quotient of $B$. Thus, without any loss of generality, we may assume that $B$ itself is antiliminary. In particular, $\overline{e B e}$ is not abelian if $e \in B$ is any nonzero projection. Write $B=\varliminf_{(\mathrm{l}}\left(D_{p}, \phi_{p+1, p}\right)$, with unital connecting maps, and with $D_{p}$ finite dimensional $\mathrm{C}^{*}$-algebras, $D_{1}=\mathbb{C}$. A system like the one in the statement will be obtained by a suitable contraction of the system $\left(D_{p}, \phi_{p+1, p}\right)$, i.e by taking $B_{n}=D_{p(n)}$ and $\psi_{n+1, n}=\phi_{p(n+1), p(n)}$ for some increasing sequence $(p(n))_{n=1}^{\infty}$. Actually it suffices to find a sequence $\left(i_{n}\right)_{n=1}^{\infty}$ such that $1 \leq i_{n} \leq t_{n}, k_{n}^{i_{n+1}, i_{n}} \geq 2$. Indeed after a suitable contraction, one may arrange that $k_{n}^{i_{n+1}, i_{n}}>k(n)$ and then relabel the blocks of $D_{p(n)}$. The construction of $p(n)$ and $i_{n}$ is done by induction. Let $p(1)=i_{1}=1$ and suppose that $p(r)$ and $i_{r}$ were constructed for $r \leq n$ such that $k_{r}^{i_{r}, i_{r-1}} \geq 2$ and 
the map $\phi_{\infty, p(n)}: D_{p(n)}^{i_{n}} \rightarrow B$ is injective. Then $p(n+1)$ and $i_{n+1}$ are obtained as follows. Let $e$ be a rank-one projection in $D_{p(n)}^{i_{n}}$. Since $\phi_{\infty, p(n)}(e) B \phi_{\infty, p(n)}(e)$ is nonzero, it cannot be abelian. Hence there is $m>p(n)$ such that the finite dimensional $\mathrm{C}^{*}$-algebra $\phi_{m, p(n)}(e) D_{m} \phi_{m, p(n)}(e)$ is not an abelian algebra. Therefore there is $i$ such that rank $\phi_{m, p(n)}^{i}(e) \geq 2$ in $D_{m}^{i}$ and the map $\phi_{\infty, m}: D_{m}^{i} \rightarrow B$ is injective. Thus we may take $p(n+1)=m$ and $i_{n+1}=i$.

If $A$ is a $\mathrm{C}^{*}$-algebra, let $C A \cong C_{0}(0,1] \otimes A$ denote the cone algebra of $A$ and let $\widetilde{C A}$ be its unitization. Note that if $A$ is RFD, then so is $\widetilde{C A}$.

Proposition 8. Let $B$ be a non type I unital AF algebra. Let $A$ be a RFD algebra, and let $\left(\gamma_{n}^{\prime}\right)_{n=1}^{\infty}$ be a strongly separating sequence of unital finite dimensional representations of $\widetilde{C A}$. Then there is a restricted Bratteli system $(\widetilde{C A}, \underline{\pi})$ such that $A F(\underline{\pi}) \cong B$ and $\gamma_{n}^{\prime}$ is a subrepresentation of $\gamma_{n}, n \geq 1$, where $\left(\gamma_{n}\right)_{n=1}^{\infty}$ is the distinguished component of $\underline{\pi}$.

Proof. Let $\gamma_{n}^{\prime}: \widetilde{C A} \rightarrow M_{k(n)}(\mathbb{C})$ be as above. Let $\left(B_{n}, \psi_{n+1, n}\right)$ be given by Lemma 7 for the input sequence $(k(n))$. Define $\pi_{n}=\left(\pi_{n}^{i, j}\right), \pi_{n}^{i, j}: \widetilde{C A} \rightarrow M_{k_{n}^{i, j}}(\mathbb{C}) \subset$ $M_{k_{n}^{i, j}}(\widetilde{C A})$, as follows. If $(i, j) \neq(1,1)$ then $\pi_{n}^{i, j}(a)=a(0) \otimes 1_{k_{n}^{i, j}}$. If $(i, j)=(1,1)$, then

$$
\pi_{n}^{1,1}(a)=\left(\begin{array}{cc}
a & 0 \\
0 & \gamma_{n}(a)
\end{array}\right)
$$

where, since $k_{n}^{1,1}>k(n)$, we can define

$$
\gamma_{n}(a)=\left(\begin{array}{cc}
\gamma_{n}^{\prime}(a) & 0 \\
0 & a(0) \otimes 1_{\ell(n)}
\end{array}\right)
$$

$\ell(n)=k_{n}^{1,1}-k(n)-1 \geq 0$.

The next result answers a question of $[\mathrm{Po}]$ as it was explained in the introduction.

Proposition 9. Let $B$ be a non type I unital separable AF algebra. Then there exists a unital quasidiagonal nonexact separable $C^{*}$-algebra $C$ with $\operatorname{RR}(C)=0$, $\operatorname{sr}(C)=1$, such that $C$ contains $B$ and is asymptotically homotopy equivalent to $B$. If $B$ has a unique unital trace, then so does $C$. Moreover $B$ and $C$ have isomorphic ideal lattices. In particular, if $B$ is simple, then $C$ is simple.

Proof. Let $A$ be a nonexact RFD $\mathrm{C}^{*}$-algebra. For instance we take $A$ to be the $\mathrm{C}^{*}$-algebra of the free group on two generators. This is an RFD $\mathrm{C}^{*}$-algebra by $[\mathrm{Ch}]$, and it is not exact [W, Corollary 3.7]. Then $\widetilde{C A}$ is not exact, since it has a nonexact quotient $([\mathrm{Ki}])$. Let $\left(\gamma_{n}^{\prime}\right)_{n=1}^{\infty}$ be a strongly separating sequence of unital finite dimensional representations of $\widetilde{C A}$. If $(\widetilde{C A}, \underline{\pi})$ is the restricted Bratteli system given by Proposition 8 , then $A F(\underline{\pi}) \cong B$, and $\left(\gamma_{n}\right)_{n=1}^{\infty}$ is a strongly separating sequence of $\widetilde{C A}$, since $\gamma_{n}^{\prime}$ is a subrepresentation of $\gamma_{n}$. Let $\kappa: \mathbb{C} \rightarrow \widetilde{C A}$ be the 
(unital) inclusion map. If we set $C=\widetilde{C A}(\underline{\pi})$, then by Remark $4, \mathbb{C}(\underline{\pi} \circ \kappa) \hookrightarrow \widetilde{C A}(\underline{\pi})$, hence

$$
B \cong A F(\underline{\pi}) \cong \mathbb{C}(\underline{\pi} \circ \kappa) \hookrightarrow \widetilde{C A}(\underline{\pi})=C .
$$

Note that $\operatorname{RR}(C)=0$ and $\operatorname{sr}(C)=1$ by Proposition 5 . Moreover $C$ is quasidiagonal since it is an inductive limit of quasidiagonal $\mathrm{C}^{*}$-algebras. By Proposition $6, C$ is not exact since $\widetilde{C A}$ is not exact. Note that $C$ is shape equivalent to $B$ since $\kappa: \mathbb{C} \rightarrow \widetilde{C A}$ is a homotopy equivalence $\left[\mathrm{B}_{2}\right]$. By $\left[\mathrm{D}_{1}\right.$, Theorem 3.9] it follows that $C$ is asymptotically homotopy equivalent to $B$. Since $\operatorname{RR}(C)=0$, the traces of $C$ are separated by the projections of $C$, hence they are determined uniquely by their action on $K_{0}(C) \cong K_{0}(B)$. Thus if $B$ has a unique unital trace, it follows that $C$ has at most one unital trace. On the other hand, $C$ is unital and quasidiagonal hence it has a unital trace by $[\mathrm{V}, 2.5]$. Finally, if $E$ is a unital $\mathrm{C}^{*}$-algebra with $\mathrm{RR}(E)=0$ and $\operatorname{sr}(E)=1$, then its two-sided closed ideals are parameterized by the order ideals of $K_{0}(E)$ (see [Z]). That proves the last part of the statement, since $K_{0}(B) \cong K_{0}(C)$ as ordered groups.

\section{Nonnuclear models of AF algebras}

Proposition 10. Let $A$ be an RFD AF algebra. Let $(\widetilde{C A}, \underline{\pi})$ be a restricted Bratteli system such that $\left(\gamma_{n}\right)_{n=1}^{\infty}$ is strongly separating for $\widetilde{C A}$. Then $\widetilde{C A}(\underline{\pi})$ is an $A F$ algebra isomorphic to $A F(\underline{\pi})$.

Proof. Let $A=\overline{\cup_{k=1}^{\infty} A_{k}}$, where $\left(A_{k}\right)$ is an increasing sequence of finite dimensional $\mathrm{C}^{*}$-algebras. Let $j_{k+1, k}: \widetilde{C A_{k}} \rightarrow \widetilde{C A_{k+1}}$ be the inclusion map. Let $\left(\widetilde{C A_{k}}, \underline{\pi}^{(k)}\right)$ be the Bratteli system obtained be restricting $\pi_{n}^{i, j}$ to $\widetilde{C A_{k}}$ (see Remark 4). Let $J_{k+1, k}: \widetilde{C A_{k}}\left(\underline{\pi}^{(k)}\right) \rightarrow \widetilde{C A_{k+1}}\left(\underline{\pi}^{(k+1)}\right)$ be the homomorphism induced by $j_{k+1, k}$. Then we have

$$
\widetilde{C A}(\underline{\pi})=\varliminf_{\longrightarrow}\left(\widetilde{C A_{k}}\left(\underline{\pi}^{(k)}\right), J_{k+1, k}\right) .
$$

Each algebra $\widetilde{C A_{k}}\left(\underline{\pi}^{(k)}\right)$ has real rank zero, trivial $K_{1}$-group, and it is an inductive limit of one dimensional noncommutative CW complexes as defined in [ELP]. Then $\widetilde{C A_{k}}\left(\underline{\pi}^{(k)}\right)$ is $\mathrm{AF}$ by [ELP, Theorem 4.2]. It follows that $\widetilde{C A}(\underline{\pi})$ is $\mathrm{AF}$ since the class of AF algebras in closed to inductive limits. Let $\kappa: \mathbb{C} \rightarrow \widetilde{C A}$ denote the inclusion map. As in Proposition $9, \kappa$ induces a unital $*$-monomorphisms $\mathbb{C}(\underline{\pi} \circ$ $\kappa) \rightarrow \widetilde{C A}(\underline{\pi})$ that is an isomorphism of scaled ordered K-theory groups since $\kappa$ is a homotopy equivalence. Therefore $A F(\underline{\pi}) \cong \mathbb{C}(\underline{\pi} \circ \kappa) \cong \widetilde{C A}(\underline{\pi})$ by Elliott's Theorem $\left(\left[\mathrm{Ell}_{1}\right]\right)$.

Proposition 10 is actually true for any nuclear RFD $\mathrm{C}^{*}$-algebra $A$. A proof of this fact is a straightforward consequence of Theorem 5 of $\left[\mathrm{D}_{3}\right]$.

Theorem 11. Let $B$ be a non type I unital AF algebra. Then $B$ contains a unital nonnuclear $C^{*}$-subalgebra $A$ with the following properties.

(a) $\operatorname{RR}(A)=0$ and $\operatorname{sr}(A)=1$. 
(b) If $\iota: A \rightarrow B$ is the inclusion map, then there is a unital *-monomorphism $\kappa: B \rightarrow A$ such that $\iota \circ \kappa$ is homotopic to $i d_{B}$ and $\kappa \circ \iota$ is asymptotically homotopic to $i d_{A}$.

In particular $K_{*}(A) \cong K_{*}(B)$ as scaled ordered groups. Therefore $A$ and $B$ have isomorphic lattices of ideals and (affinely) homeomorphic trace spaces.

Proof. First we construct a non type I RFD AF algebra. Let $L$ be a non type I AF algebra, for instance the CAR algebra. Write $L=\overline{\cup_{k=1}^{\infty} L_{k}}$, where $\left(L_{k}\right)$ is an increasing sequence of finite dimensional $C^{*}$-algebras. Let $\mathbb{N} \cup\{\infty\}$ be the one point compactification of $\mathbb{N}$ and let $D$ be the $\mathrm{C}^{*}$-algebra of all continuous functions $f: \mathbb{N} \cup\{\infty\} \rightarrow L$ such that $f(k) \in L_{k}$ for all $k \in \mathbb{N}$. Then $D$ is an RFD AF algebra. It is not type $\mathrm{I}$ as it has $L$ as a quotient. Therefore by [ $\mathrm{B}_{1}$, Theorem 1$], D$ contains a nonnuclear $C^{*}$-subalgebra $E$. Using Proposition 8 for $B$ and the RFD algebra $D$, we find a restricted Bratteli system $(\widetilde{C D}, \underline{\pi})$ such that its distinguished component $\left(\gamma_{n}\right)_{n=1}^{\infty}$ is a strongly separating sequence of unital finite dimensional representations of $\widetilde{C D}$, and $B \cong A F(\underline{\pi})$. Let $\iota_{0}: \widetilde{C E} \rightarrow \widetilde{C D}$ be the (unital) inclusion map. By Remark 4, $\iota_{0}$ induces a $*$-monomorphism $\iota: \widetilde{C E}\left(\underline{\pi} \circ \iota_{0}\right) \rightarrow$ $\widetilde{C D}(\underline{\pi})$. We let $A=\widetilde{C E}\left(\underline{\pi} \circ \iota_{0}\right)$ and note that $A$ is not nuclear by Proposition 6 . By Proposition $10, \widetilde{C D}(\underline{\pi})$ is isomorphic to $A F(\underline{\pi}) \cong B$, so that we may identify these algebras. Note that now (a) is a consequence of Proposition 5. The proof of (b) is more involved. One can see that since $\iota_{0}$ is a homotopy equivalence, $A$ is shape equivalent to $B$, hence $A$ is asymptotically homotopy equivalent to $B$ by $\left[\mathrm{D}_{1}\right.$, Theorem 3.14]. While this proves the assertion about K-theory, it does not prove part (b). One needs to show that the maps implementing the asymptotic homotopy are genuine *-homomorphisms. This is achieved by using the following two claims whose proofs are given later.

Claim 1. There is a unit preserving asymptotic morphism $\left(\kappa_{t}\right): B \rightarrow A$ such that $\left(\kappa_{t}\right) \circ \iota$ is asymptotically homotopic to $i d_{A}$.

Claim 2. Any unital asymptotic morphism from $B$ to $A$ is (asymptotically) homotopic to a unital $*$-homomorphism.

If $\left(\varphi_{t}\right),\left(\psi_{t}\right)$ are asymptotic morphisms we write $\left(\varphi_{t}\right) \sim\left(\psi_{t}\right)$ if the two asymptotic morphisms are homotopic. Let $\left(\kappa_{t}\right)$ be given by Claim 1. Then $\left(\kappa_{t}\right) \circ \iota \sim i d_{A}$. By Claim 2, there is a unital $*$-homomorphism $\kappa: B \rightarrow A$ such that $\left(\kappa_{t}\right) \sim \kappa$. Thus $\kappa \circ \iota \sim i d_{A}$. It remains to prove that $\iota \circ \kappa \sim i d_{B}$ and that $\kappa$ is injective. To that purpose, note that $\iota_{*}: K_{*}(A) \rightarrow K_{*}(B)$ is an isomorphism since $\iota_{0}$ is a homotopy equivalence. Therefore $\kappa_{*}=\left(\iota_{*}\right)^{-1}$, since $\kappa_{*} \circ \iota_{*}=\left(i d_{A}\right)_{*}$, so that $\kappa$ is injective and $\iota_{*} \circ \kappa_{*}=\left(i d_{B}\right)_{*}$. That implies that $\iota \circ \kappa$ is homotopic to $i d_{B}$, since $B$ is an AF algebra, by an argument similar to one in $\left[\mathrm{B}_{3}, 7.7 .5\right]$.

Next we discuss the last part of the Theorem. If $C$ is a unital $\mathrm{C}^{*}$-algebra, let $T(C)$ denote the space of unital traces of $C$ and let $S K_{0}(C)$ denote the set of all positive group homomorphisms $f: K_{0}(C) \rightarrow \mathbb{R}$ with $f\left[1_{C}\right]=1$. If $R R(C)=0$, then the natural map $T(C) \rightarrow S K_{0}(C)$ is injective. It is also surjective if $C$ is $\mathrm{AF}\left(\left[\mathrm{B}_{3}\right]\right)$. It follows that the restriction map $T(B) \rightarrow T(A)$ is bijective (hence an homeomorphism in the weak* topology), since $K_{0}(A) \cong K_{0}(B)$ as ordered scaled 
groups. As in the proof of Proposition 9, the two-sided closed ideals are in bijection with the order ideals of the $K_{0}$-group.

The proofs of the two claims above require some preparation. Let $\mathcal{A}$ be the asymptotic homotopy category of Connes and Higson $[\mathrm{CH}]$. Its objects are separable $\mathrm{C}^{*}$-algebras and its morphisms are homotopy classes of asymptotic morphisms. One can construct asymptotic morphisms using the homotopy inductive limit functor $L$ of $\left[\mathrm{D}_{1}\right] . L$ is defined on the strong shape category with values in $\mathcal{A}$. It takes an inductive system of $\mathrm{C}^{*}$-algebras $\left(C_{n}, p_{n+1, n}\right)$ to its $\operatorname{limit} \underline{\lim }\left(C_{n}, p_{n+1, n}\right)$. To describe the action of $L$ on morphisms, recall that a strong map of (inductive) systems

$$
(\underline{f}, \underline{h}):\left(C_{n}, p_{n+1, n}\right) \rightarrow\left(D_{n}, q_{n+1, n}\right)
$$

can be given by a sequence of $*$-homomorphisms $f_{n}: C_{n} \rightarrow D_{n}$ and by a sequence of homotopies $h_{n}=\left(h_{n}^{t}\right): C_{n} \rightarrow D_{n+1}[0,1]$ such that $h_{n}^{0}=q_{n+1, n} f_{n}$ and $h_{n}^{1}=f_{n+1} p_{n+1, n}$. Let $p_{k, n}: C_{n} \rightarrow C_{k}$ denote the connecting map. Let $D=\lim _{(}\left(D_{n}, q_{n+1, n}\right)$ and let $q_{n}: D_{n} \rightarrow D$ be the canonical map. Then $L(f, \underline{h})$ is an asymptotic morphism $\left(\varphi_{t}\right)$ whose construction goes as follows. Let $T_{n}=[n, \infty)$ and for every $n$ define $*$-homomorphisms $\varphi_{n}: C_{n} \rightarrow C_{b}\left(T_{n}, D\right)$ by

$$
\varphi_{n}^{t}=q_{k+1} h_{k}^{t-k} p_{k, n}, \quad n \leq k \leq t \leq k+1 .
$$

Let $r_{n+1, n}: C_{b}\left(T_{n}, D\right) \rightarrow C_{b}\left(T_{n+1}, D\right)$ be the restriction map. The $*$-homomorphisms $\varphi_{n}$ fit together in a commutative diagram.

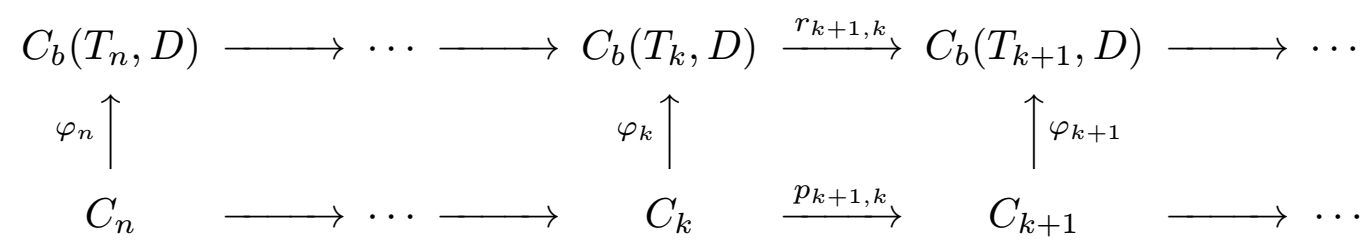

Passing to inductive limit we obtain a $*$-homomorphism

$$
\hat{\varphi}=\underline{\lim _{n}} \varphi_{n}: C=\underline{\lim _{\longrightarrow}}\left(C_{n}, p_{n+1, n}\right) \rightarrow \varliminf_{\lim _{b}} C_{b}\left(T_{n}, D\right)=C_{b}\left(\mathbb{R}_{+}, D\right) / C_{0}\left(\mathbb{R}_{+}, D\right) .
$$

Let $\varphi=\left(\varphi_{t}\right): C \rightarrow C_{b}\left(\mathbb{R}_{+}, D\right)$ be a continuous lifting of $\hat{\varphi}$. Then $L(\underline{f}, \underline{h})=\left(\varphi_{t}\right)$ is defined up to an equivalence of asymptotic morphisms. There is an appropriate notion of homotopy for strong maps of systems such that $L$ descends to a well defined map on homotopy classes. A rather general example of a homotopy of strong maps $(f, \underline{h}),\left(f^{\prime}, \underline{h}^{\prime}\right):\left(C_{n}, p_{n+1, n}\right) \rightarrow\left(D_{n}, q_{n+1, n}\right)$ is given by a strong map of systems $(\underline{\nu}, \underline{\mu}):\left(\bar{C}_{n}, p_{n+1, n}\right) \rightarrow\left(D_{n}[0,1], q_{n+1, n} \otimes i d_{C[0,1]}\right)$ where $\underline{\nu}=\left(\nu^{s}\right)_{s \in[0,1]}$ and $\underline{\mu}=\left(\mu^{s}\right)_{s \in[0,1]}$ are such that $\left(\underline{\nu}^{0}, \underline{\mu}^{0}\right)=(\underline{f}, \underline{h})$ and $\left(\underline{\nu}^{1}, \underline{\mu}^{1}\right)=\left(\underline{f}^{\prime}, \underline{h}^{\prime}\right)$. More explicitly, for every $n,\left(\nu_{n}^{s}\right): C_{n} \rightarrow D_{n}$ is a homotopy of $*$-homomorphisms with $\nu_{n}^{0}=f_{n}$ and $\nu_{n}^{1}=f_{n}^{\prime}$ while $\mu_{n}^{s, t}: C_{n} \rightarrow D_{n+1}$ is a two-parameter homotopy of $*$-homomorphisms such that for all $0 \leq s, t \leq 1$

$$
\begin{aligned}
& \mu_{n}^{s, 0}=q_{n+1, n} \nu_{n}^{s} \\
& \mu_{n}^{s, 1}=\nu_{n+1}^{s} p_{n+1, n} \\
& \mu_{n}^{0, t}=h_{n}^{t} \\
& \mu_{n}^{1, t}=h_{n}^{\prime t} .
\end{aligned}
$$


If $(\underline{\nu}, \underline{\mu})$ is as above, then $L(\underline{\nu}, \mu)$ is a homotopy of asymptotic morphisms from $L((\underline{f}, \underline{h}))$ to $L\left(\left(\underline{f}^{\prime}, \underline{h}^{\prime}\right)\right)$ (see $\left.\left[\mathrm{D}_{1}\right]\right)$.

Claim 2 is a consequence of the following Lemma.

Lemma 12. Let $B$ be an $A F$ algebra and let $A$ be a unital $C^{*}$-algebra. Then every asymptotic morphism $\left(\varphi_{t}\right): B \rightarrow A$ is homotopic to a $*$-homomorphism.

Proof. Write $B=\varliminf_{\longrightarrow}\left(B_{n}, p_{n+1, n}\right)$ with $B_{n}$ finite dimensional $\mathrm{C}^{*}$-algebras. The system $\left(B_{n}, p_{n+1, n}\right)$ is semiprojective $\left[\mathrm{B}_{2}\right],\left[\mathrm{D}_{1}\right.$, Def. 3.2]. Then as in $\left[\mathrm{D}_{1}\right.$, Corollary $3.14]$ we can lift $\left(\varphi_{t}\right)$ to a strong map of systems $\left(\underline{f}^{\prime}, \underline{h}^{\prime}\right):\left(B_{n}, p_{n+1, n}\right) \rightarrow(A, i d)$

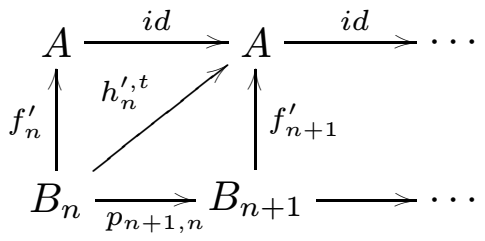

with $h_{n}^{\prime}: B_{n} \rightarrow A[0,1]$ satisfying $h_{n}^{\prime 0}=f_{n}^{\prime}$ and $h_{n}^{\prime 1}=f_{n+1}^{\prime} p_{n+1, n}$. We will construct inductively a sequence of unitaries $\left(z_{n}\right)$ in $A$ such that if we define $f_{n}=a d\left(z_{n}\right) f_{n}^{\prime}$, then $f_{n+1} p_{n+1, n}=f_{n}$ and if we set $h_{n}^{t}=f_{n}$, then $(\underline{f}, \underline{h})$ is homotopic to $\left(\underline{f}^{\prime}, \underline{h}^{\prime}\right)$ as strong maps of systems via some homotopy $(\underline{\nu}, \underline{\mu})$. That will imply that the *-homomorphism $\underline{\lim }_{f_{n}}=L(\underline{f}, \underline{h})$ is homotopic to $L\left(\underline{f}^{\prime}, \underline{h}^{\prime}\right) \sim\left(\varphi_{t}\right)$.

The homotopies $\nu_{n}^{s}$ and $\mu_{n}^{s, t}$ are constructed by induction and such that $\nu_{n}^{s}=$ $a d\left(u_{n}^{s}\right) f_{n}^{\prime}$ where $\left(u_{n}^{s}\right)$ is a path of unitaries in $A$ with $u_{n}^{0}=z_{n}$ and $u_{n}^{1}=1$. The first step is trivial as we can assume that $B_{1}=B_{2}=\mathbb{C}$. Suppose now that $z_{k}, u_{k}^{s}, \nu_{k}^{s}$ and $\mu_{k-1}^{s, t}$ were constructed for $k \leq n$. Then $z_{n+1}, u_{n+1}^{s}, \nu_{n+1}^{s}$ and $\mu_{n}^{s, t}$ are constructed as follows. We regard $h_{n}^{\prime t}$ as a continuous path into the path-connected component of $f_{n}^{\prime}$ in the base space of the fibration $U(A) \rightarrow \operatorname{Hom}\left(B_{n}, A\right)$ (see $\left.[\mathrm{N}]\right)$. Therefore we can find a continuous path of unitaries $\left(w_{n}^{t}\right)$ in $A$ such that $w_{n}^{0}=1$ and $h_{n}^{\prime t}=\operatorname{ad}\left(w_{n}^{t}\right) f_{n}^{\prime}$. Let $\left(v_{n}^{s}\right)$ be a path given by the concatenation $\left(u_{n}^{s}\right) *\left(w_{n}^{s}\right)$. Then there exists a continuous map $\omega:[0,1] \times[0,1] \rightarrow U(A)$ such that

$$
\omega(s, 0)=u_{n}^{s}, \omega(s, 1)=v_{n}^{s}, \omega(0, t)=u_{n}^{0}, \omega(1, t)=w_{n}^{t} .
$$

The map $\omega$ exists as its prescribed restriction to the boundary of the unit square gives a trivial element of the fundamental group of $U(A)$, by our choice of $v_{n}^{s}$. Define

$$
\begin{gathered}
u_{n+1}^{s}=v_{n}^{s} w_{n}^{1 *}, z_{n+1}=u_{n+1}^{0} \\
\nu_{n+1}^{s}=\operatorname{ad}\left(u_{n+1}^{s}\right) f_{n+1}^{\prime} \quad \mu_{n}^{s, t}=\operatorname{ad}(\omega(s, t)) f_{n}^{\prime} .
\end{gathered}
$$

It is then easy to check that $(\underline{\nu}, \underline{\mu})$ gives the desired homotopy, as it satisfies $(4)$.

To complete the proof of Theorem 11 it remains to prove Claim 1. 
The asymptotic morphism $\left(\kappa_{t}\right): \widetilde{C D}(\underline{\pi}) \rightarrow \widetilde{C E}(\underline{\pi})$ will be constructed by using the following strong map of systems.

$$
(\underline{\kappa}, \underline{q}):\left(\bigoplus_{i=1}^{t_{n}} M_{[n, i]}(\widetilde{C D}), \phi_{n}\right) \rightarrow\left(\bigoplus_{i=1}^{t_{n}} M_{[n, i]}(\widetilde{C E}), \phi_{n}\right)
$$

where $\kappa_{n}(a)=a(0)$ and $q_{n}^{t}=\left(i d_{[n, j]} \otimes q_{n}^{i, j, t}\right), q_{n}^{i, j, t}: \widetilde{C D} \rightarrow M_{k_{n}^{i, j}}(\mathbb{C}) \subset M_{k_{n}^{i, j}}(\widetilde{C E})$, $q_{n}^{i, j, t}(a)=\pi_{n}^{i, j}(a(t \cdot))$ if $(i, j) \neq(1,1)$, and

$$
q_{n}^{1,1, t}(a)=\left(\begin{array}{cc}
a(0) & 0 \\
0 & \gamma_{n}(a(t \cdot))
\end{array}\right)
$$

The $*$-homomorphism $\iota: \widetilde{C E} \rightarrow \widetilde{C D}$ is induced by the following strong map of systems.

$$
(\underline{\iota}, \underline{g}):\left(\bigoplus_{i=1}^{t_{n}} M_{[n, i]}(\widetilde{C E}), \phi_{n}\right) \rightarrow\left(\bigoplus_{i=1}^{t_{n}} M_{[n, i]}(\widetilde{C D}), \phi_{n}\right)
$$

where $\iota_{n}$ is induced by the inclusion $\widetilde{C E} \hookrightarrow \widetilde{C D}$, and $g_{n}^{t}=\phi_{n} \iota_{n}$ is a constant homotopy. We have that

$$
(\underline{\kappa}, \underline{q}) \circ(\underline{\iota}, \underline{g})=(\underline{f}, \underline{h})
$$

where

$$
(\underline{f}, \underline{h}):\left(\bigoplus_{i=1}^{t_{n}} M_{[n, i]}(\widetilde{C E}), \phi_{n}\right) \rightarrow\left(\bigoplus_{i=1}^{t_{n}} M_{[n, i]}(\widetilde{C E}), \phi_{n}\right),
$$

$f_{n}(a)=a(0)$ and $h_{n}^{t}=\left(i d_{[n, j]} \otimes h_{n}^{i, j, t}\right), h_{n}^{i, j, t}: \widetilde{C E} \rightarrow M_{k_{n}^{i, j}}(\widetilde{C E})$, $h_{n}^{i, j, t}(a)=\pi_{n}^{i, j}(a(t \cdot))$ if $(i, j) \neq(1,1)$, and

$$
h_{n}^{1,1, t}(a)=\left(\begin{array}{cc}
a(0) & 0 \\
0 & \gamma_{n}(a(t \cdot))
\end{array}\right)
$$

We are going to prove that $(\underline{f}, \underline{h})$ is homotopic to $\left(\underline{f}^{\prime}, \underline{h}^{\prime}\right)$ as strong maps of systems, where $f_{n}^{\prime}=i d_{\oplus_{i} M_{[n, i]}(\widetilde{C E})}$ and $h_{n}^{\prime t}=\phi_{n}$ is a constant homotopy. To that purpose define a homotopy $(\underline{\nu}, \underline{\mu})$ by $\nu_{n}^{s}(a)=a(s \cdot)$ and $\mu_{n}^{s, t}=\left(i d_{[n, j]} \otimes \mu_{n}^{i, j, s, t}\right)$, $\mu^{i, j, s, t}: \widetilde{C E} \rightarrow M_{k_{n}^{i, j}}(\widetilde{C E}), \mu^{i, j, s, t}(a)=\pi_{n}^{i, j}(a(\max (s, t) \cdot))$ if $(i, j) \neq(1,1)$, and

$$
\mu^{1,1, s, t}(a)=\left(\begin{array}{cc}
a(s \cdot) & 0 \\
0 & \gamma_{n}(a(\max (s, t) \cdot))
\end{array}\right)
$$

Then $(\underline{\nu}, \underline{\mu})$ will give a homotopy from $(\underline{f}, \underline{h})$ to $\left(\underline{f}^{\prime}, \underline{h}^{\prime}\right)$, since it satisfies $(4)$. Finally let us notice that if we set $\left(\kappa_{t}\right)=L(\underline{\kappa}, \underline{q})$, then since $L(\underline{\iota}, \underline{g}) \sim \iota$, and $L\left(\underline{f}^{\prime}, \underline{h}^{\prime}\right)=i d_{A}$, we have

$$
\left(\kappa_{t}\right) \circ \iota \sim L(\underline{\kappa}, \underline{q}) \circ L(\underline{\iota}, \underline{g}) \sim L(\underline{f}, \underline{h}) \sim L\left(\underline{f}^{\prime}, \underline{h}^{\prime}\right) \sim i d_{A}
$$


That proves Claim 1 and completes the Proof of Theorem 11.

\section{Nonisomorphic exact $\mathrm{C}^{*}$-algebras}

Note that the $\mathrm{C}^{*}$-algebras $A, B$ of Theorem 11 have the same Elliott invariants. They are not isomorphic since $A$ is not nuclear whereas $B$ is nuclear.

The following example is joint work with Chris Phillips.

Example 13. There are two nonisomorphic separable unital $\mathrm{C}^{*}$-algebras, $E$ and $F$, such that

(i) $E$ and $F$ are simple, exact, nonnuclear, and have a unique unital trace.

(ii) $R R(E)=R R(F)=0$ and $\operatorname{sr}(E)=\operatorname{sr}(F)=1$.

(iii) $K_{0}(E) \cong K_{0}(F)$ as ordered scaled groups and $K_{1}(E) \cong K_{1}(F)$.

(iv) $E$ and $F$ have the same Haagerup invariant, equal to 1.

$E$ is not isomorphic to $F$ since $E$ is quasidiagonal, whereas $F$ is not. Actually $E \otimes K(H)$ is not asymptotically homotopy equivalent (via completely positive asymptotic morphisms) to $F \otimes K(H)$ since quasidiagonality is invariant under such an equivalence (see $\left.\left[\mathrm{D}_{2}\right]\right)$.

Let $B$ be the UHF algebra of type $2^{\infty}$. Let $D$ be a simple real rank zero $A \mathbb{T}$ algebra, with $K_{0}(D)=\mathbb{Z}[1 / 2]$ (with natural order), $\left[1_{D}\right]=1$, and $K_{1}(D)=\mathbb{Z}^{2}$ $\left(\left[\mathrm{Ell}_{2}\right]\right)$. Let $A \subset B$ be given by Theorem 11. We set $E=B \otimes A \otimes D$ and $F=B \otimes A \otimes C_{r}^{*}\left(\mathbb{F}_{2}\right)$, where all tensor products are minimal. Recall that the reduced $\mathrm{C}^{*}$-algebra of the free group on two generators, $C_{r}^{*}\left(\mathbb{F}_{2}\right)$, is simple and has a unique unital trace [Pow]. Next we verify the properties of $E$ and $F$ listed above.

(i) $E$ and $F$ are simple and exact since they are minimal tensor products of simple, exact $\mathrm{C}^{*}$-algebras $\left(\left[\mathrm{Ta}_{2}, \mathrm{Ch} \mathrm{IV}, 4.21\right],[\mathrm{W}]\right)$. They have a unique unital trace since each factor in the tensor product has a unital unique trace $[\mathrm{CuP}$, Cor. 6.13]. Using a slice map, one shows that a (nonzero) minimal tensor product by a nonnuclear $\mathrm{C}^{*}$-algebra is nonnuclear. Thus both $E$ and $F$ are nonnuclear.

(ii) As in (i), $E_{1}=A \otimes D$ and $F_{1}=A \otimes C_{r}^{*}\left(\mathbb{F}_{2}\right)$ are simple with unique unital trace. It follows that $E=B \otimes E_{1}$ and $F_{1}=B \otimes F_{1}$ have stable rank one by [R $\left.\varnothing_{1}\right]$ and real rank zero by $\left[\mathrm{R} \emptyset_{2}\right.$, Theorem 7.2] and $\left[\mathrm{H}_{3}\right.$, Theorem 5.11].

(iii) We have $B \otimes B \cong B$. Recall that shape equivalence is preserved under taking minimal tensor products $\left[\mathrm{B}_{2}\right]$, and that $A$ is shape equivalent to $B$. Thus $E$ is shape equivalent to $B \otimes D$ and $F$ is shape equivalent to $B \otimes C_{r}^{*}\left(\mathbb{F}_{2}\right)$. By [PiV], and the Künneth formula $([\mathrm{Sc}])$, we see that $K_{*}(B \otimes D) \cong K_{*}\left(B \otimes C_{r}^{*}\left(\mathbb{F}_{2}\right)\right)$ hence $K_{*}(E) \cong K_{*}(F)$. It remains to show that the positive cones $K_{0}(-)^{+}$are isomorphic. First we see that $K_{0}(B \otimes D)^{+} \cong \mathbb{Z}[1 / 2]^{+}$by construction. The isomorphism $K_{0}(B \otimes$ $\left.C_{r}^{*}\left(\mathbb{F}_{2}\right)\right)^{+} \cong \mathbb{Z}[1 / 2]^{+}$follows from the fact that any projection in $M_{n}\left(C_{r}^{*}\left(\mathbb{F}_{2}\right)\right)$ is equivalent to $1_{k}$, for some $k \leq n$, since $K_{0}\left(C_{r}^{*}\left(\mathbb{F}_{2}\right)\right)=\mathbb{Z}$ is generated by the class of 1 , by $[\mathrm{PiV}]$, and since $C_{r}^{*}\left(\mathbb{F}_{2}\right)$ has stable rank one by [DyHR $\left.\varnothing\right]$.

(iv) Let $\Lambda$ denote the Haagerup invariant. We have that $\Lambda=1$ for nuclear $\mathrm{C}^{*}$ algebras and $\Lambda\left(C_{r}^{*}\left(\mathbb{F}_{2}\right)\right)=1$ by $\left[\mathrm{H}_{1,2}\right]$. Therefore $\Lambda(E)=\Lambda(F)$ since $\Lambda(X \otimes Y)=$ $\Lambda(X) \Lambda(Y)$, for (exact) $\mathrm{C}^{*}$-algebras $X, Y$ and minimal tensor products ([SiSm $]$ ). 
Example of simple exact nonisomorphic $\mathrm{C}^{*}$-algebras (of real rank one) with the same Elliott and Haagerup invariants have recently been exhibited in [GePh].

\section{REFERENCES}

[B $\left.\mathrm{B}_{1}\right] \quad$ B. Blackadar, Nonnuclear subalgebras of $C^{*}$-algebras, J. Operator Theory 14 (1985), 347-350.

$\left[\mathrm{B}_{2}\right] \quad$ B. Blackadar, Shape theory for $C^{*}$-algebras, Math. Scand. 56 (1985), 249-275.

$\left[\mathrm{B}_{3}\right] \quad$ B. Blackadar, K-theory for Operator Algebras, M. S. R. I. Monographs No. 5, SpringerVerlag, Berlin and New York, 1986.

[Br] O. Bratteli, Inductive limits of finite-dimensional $C^{*}$-algebras, Trans. Amer. Math. Soc. 171 (1972), 195-234.

[BP] L. G. Brown and G. K. Pedersen, $C^{*}$-algebras of real rank zero, J. Funct. Anal. 99 (1991), 131-149.

[C] Alain Connes, Noncommutative Geometry, Academic Press, New York, London, 1994.

$[\mathrm{CH}] \quad$ A. Connes and N. Higson, Deformations, morphismes asymptotiques et $K$-theorie bivariante, C.R. Acad. Sci. Paris 313 (1990).

[Ch] M. -D. Choi, The full $C^{*}$-algebra of the free group on two generators, Pacific J. Math 87 (1980), 41-48.

[ChE] M. -D. Choi and E. G. Effros, The completely positive lifting problem for $C^{*}$-algebras, Ann. of Math. 104 (1976), 585-609.

[CuP] J. Cuntz and G. K. Pedersen, Equivalence and traces on $C^{*}$-algebras, J. Funct. Anal. 33 (1979), 135-164.

[D 1 M. Dadarlat, Shape theory and asymptotic morphisms for $C^{*}$-algebras, Duke Math. J. 73 (1994), 687-711.

$\left[\mathrm{D}_{2}\right] \quad$ M. Dadarlat, Quasidiagonal morphisms and homotopy, J. Funct. Anal. 151 (1997), 231-233.

$\left[\mathrm{D}_{3}\right] \quad$ M. Dadarlat, Residually finite-dimensional $C^{*}$-algebras, Operator algebras and operator theory (Shanghai, 1997) Contemp. Math, vol. 228, Amer. Math. Soc., Providence, RI, 1998, pp. 45-50.

[DL] M. Dadarlat and T. A. Loring, The K-theory of abelian subalgebras of AF algebras, J. reine angew. Math. 432 (1992), 39-55.

[DyHRø] K. J. Dykema, U. Haagerup and M. Rørdam, The stable rank of some free product $C^{*}$-algebras, Duke Math. J. 90 (1997), 95-121.

[ELP] S. Eilers, T. A. Loring and G.K. Pedersen, Fragility for subhomogeneous $C^{*}$-algebras with one-dimensional spectrum, Bull. London Math. Soc. 31 (1999), no. 3, 337-344.

[EK] E. G. Effros and J. Kaminker, Homotopy continuity and shape theory for $C^{*}$-algebras, Geometric methods in operator algebras, US-Japan seminar at Kyoto 1983, Pitman Res. Notes Math. Ser., vol. 123, Pitman, 1986.

$\left[\mathrm{Ell}_{1}\right] \quad$ G. A. Elliott, On the classification of inductive limits of sequences of semisimple finitedimensional algebras, J. Algebra 38 (1976), 29-44.

$\left[\mathrm{Ell}_{2}\right] \quad$ G. A. Elliott, On the classification of $C^{*}$-algebras of real rank zero I, J. reine angew. Math. 443 (1993), 179-219.

[Go] K. Goodearl, Notes on a class of simple $C^{*}$-algebras with real rank zero, Publ. Math. 36 (1992), 637-654.

[GePh] L. Ge and N. C. Phillips, Nonisomorphic simple exact $C^{*}$-Algebras with the same Elliott and Haagerup invariants, in preparation.

$\left[\mathrm{H}_{1}\right] \quad \mathrm{U}$. Haagerup, An example of a nonnuclear $C^{*}$-algebra which has the metric approximation property, Invent. Math 50 (1979), 279-293.

$\left[\mathrm{H}_{2}\right] \quad$ U. Haagerup, Group $C^{*}$-algebras without the completely bounded approximation property, Preprint 1988.

$\left[\mathrm{H}_{3}\right] \quad$ U. Haagerup, Quasitraces on exact $C^{*}$-algebras are traces, Manuscript 1991. 
[Ha] D. Hadwin, Strongly quasidiagonal $C^{*}$-algebras, J. Operator Th. 18 (1987), 3-15.

[Ki] E. Kirchberg, Exact $C^{*}$-algebras, Tensor products, and Classification of purely infinite algebras, Proc. ICM Zurich 1994, Birkhäuser, Basel, 1995, pp. 943-954.

[N] V. Nistor, On the homotopy groups of the automorphism group of AF algebras, J. Operator Th. 19 (1988), 319-340.

[Pe] G. K. Pedersen, $C^{*}$-algebras and their automorphism groups, Academic Press, London, 1979.

[PiV] M. Pimsner and D. Voiculescu, Exact sequences for K-groups and Ext-groups of certain cross-products of $C^{*}$-algebras, J. Operator Theory 4 (1980), 93-111.

[Po] S. Popa, On local finite dimensional approximation of $C^{*}$-algebras, Pacific J. Math. 181 (1997), 141-158.

[Pow] R. T. Powers, Simplicity of the $C^{*}$-algebra associated with the free group on two, Duke Math. J. (1975), 151-156.

[Ri] M. A. Rieffel, Dimension and stable rank in the K-theory of $C^{*}$-algebras, Pr oc. London Math. Soc. 46 (1983), 301-333.

[R] J. Rosenberg, Quadiagonality and Nuclearity, Appendix to Strongly quasidiagonal C*algebras by D. Hadwin, J. Operator Th. 18 (1987), 15-18.

$\left[\mathrm{R} \varnothing_{1}\right] \quad$ M. Rørdam, On the structure of simple $C^{*}$-algebras tensored with a UHF algebra I, J. Funct. Anal. 101 (1991), 1-17.

[Rø, $\quad$ M. Rørdam, On the structure of simple $C^{*}$-algebras tensored with a UHF algebra II, J. Funct. Anal. 107 (1992), 255-269.

[SiSm] A. M. Sinclair and R. Smith, The Haagerup invariant for tensor products of operator spaces, Math. Proc. Cambridge Philos. Soc. 120 (1996), 147-153.

[Sc] Topological methods for $C^{*}$-algebras. II. Geometry resolutions and the Künneth formula, Pacific J. Math. (1982), 443-458.

[Ta 1 M. Takesaki, On the cross-norm of the direct product of $C^{*}$-algebras, Tŏhoku Math. J. 15 (1964), 111-122.

[Ta 2 M. Takesaki, Theory of operator algebras. I., Springer-Verlag, New York-Heidelberg, 1979.

[V] D. Voiculescu, Around quasidiagonal operators, Integr. Equat. Oper. Th. 17 (1993), 137-149.

[W] S. Wassermann, Exact $C^{*}$-algebras and related topics, Lect. Notes Ser. N. 19, Seoul Nat. Univ., Seoul.

[Zh] S. Zhang, A Riesz decomposition property and ideal structure of multiplier algebras, J. Operator Th. 24 (1990), 209-225.

Department of Mathematics, Purdue University, West Lafayette, IN 47907 USA

E-mail address: mdd@math.purdue.edu 